\title{
Mechanical Characterization of Torsional Micropaddles Using Atomic Force Microscopy
}

\author{
N. Mahmoodi (iD, ${ }^{1}$ A. Sabouri, ${ }^{1}$ J. Bowen, ${ }^{2}$ C. J. Anthony, ${ }^{1}$ and P. M. Mendes ${ }^{3}$ \\ ${ }^{1}$ Department of Mechanical Engineering, University of Birmingham, Birmingham B15 2TT, UK \\ ${ }^{2}$ School of Engineering and Innovation, The Open University, Milton Keynes MK7 6AA, UK \\ ${ }^{3}$ School of Chemical Engineering, University of Birmingham, Birmingham B15 2TT, UK
}

Correspondence should be addressed to N. Mahmoodi; nasim.mahmoodi0@gmail.com

Received 28 February 2018; Revised 25 May 2018; Accepted 20 June 2018; Published 7 August 2018

Academic Editor: Vincenzo Spagnolo

Copyright (c) 2018 N. Mahmoodi et al. This is an open access article distributed under the Creative Commons Attribution License, which permits unrestricted use, distribution, and reproduction in any medium, provided the original work is properly cited.

The reference cantilever method is shown to act as a direct and simple method for determination of torsional spring constant. It has been applied to the characterization of micropaddle structures similar to those proposed for resonant functionalized chemical sensors and resonant thermal detectors. It is shown that this method can be used as an effective procedure to characterize a key parameter of these devices and would be applicable to characterization of other similar MEMS/NEMS devices such as micromirrors. In this study, two sets of micropaddles are manufactured (beams at centre and offset by $2.5 \mu \mathrm{m}$ ) by using LPCVD silicon nitride as a substrate. The patterning is made by direct milling using focused ion beam. The torsional spring constant is achieved through micromechanical analysis via atomic force microscopy. To obtain the gradient of force curve, the area of the micropaddle is scanned and the behaviour of each pixel is investigated through an automated developed code. The experimental results are in a good agreement with theoretical results.

\section{Introduction}

MEMS devices utilizing rotation of surfaces controlled by torsional springs have gained a lot of interest. Their use as micromirrors has led to the rapid advances in digital light processing [1]; they have been shown to act as thermal infrared detectors $[2,3]$ and also as resonant mass sensing devices $[4,5]$. A resonant micropaddle is one such resonant torsional system and has been considered as a potential bio/chemical sensor platform $[6,7]$. It can detect induced mass adsorption on its surface by measuring the frequency shift of the resonance, using the so-called mass loading effect. The first resonance mode of the supported micropaddle is torsional where the beams act like a torsional spring and the paddle rotates about this axis. Although the torsional spring constant can be calculated analytically and modelled through finite element methods, it is essential to have experimental verification of such a key parameter of the device. Experimentally, measurement of the torsional spring constant can lead to a better understanding of the mechanical behaviour of the sensor. The field of atomic force microscopy is well versed in the calibration of spring constants, albeit for cantilevers, and so it is to this field that the authors have turned for methods which apply to torsional systems.

The importance of the AFM probe's spring constant for measuring the interaction force between the probe and sample has been recognized since its invention in 1986 [8]. The determination of probe spring constants dates back to the 1990s [9-13]. Common methods for the determination of cantilever probe spring constant are based on calculation from dimension and material properties [14], the use of a reference cantilever $[15,16]$, added mass $[9,17]$, and thermal noise analysis $[18,19]$.

Calculation based on cantilever dimension and modulus of elasticity is not a precise method as the dimensions are not well known and modulus of elasticity can vary from one batch to another due to different deposition conditions, specific treatment, and so forth [20]. In the added mass technique, the spring constant of a cantilever can be determined based on shift in resonance frequency due to added mass and is dependent on the effective mass and resonant frequency before and after adding the mass [9]. Spring constant 


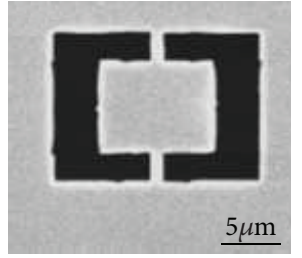

(a)

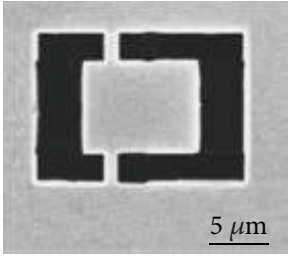

(b)
FIgURE 1: SEM images of the micropaddle with (a) anchors at the centre (MP-1) and (b) anchors offset from the centre by $2.5 \mu \mathrm{m}$ (MP-2).

of a cantilever can also be evaluated by using its length, width, resonance frequency, and material properties without knowing the exact thickness value [21]. All of these methods have uncertainty in the result which makes device performance predictions difficult; that is, in thermal noise analysis, the thermal noise in the probe deflection is related to its spring constant using equipartition theorem [20] and so ultimate resolution is innately linked to accurate knowledge of spring constants.

The reference cantilever method was first proposed by Torri et al. [12] and further developed by Tortonese and Kirk [13]. In this method, a cantilever with unknown spring constant is pushed against a reference cantilever with known spring constant. The spring constant using this method can be evaluated based on the reference cantilever spring constant and the gradient of the force curve obtained during the measurement. The use of AFM as the reference cantilever has the added advantage that the unknown cantilever can be scanned prior to being pushed and the reference cantilever can be precisely positioned, as spring constant is dependent on the position of the applied force from the anchor.

It is this reference cantilever method, proposed by Torii et al. [12], that has been investigated in this work for its applicability to measuring torsional spring constants. The manuscript presents the fabrication of two micropaddle structures, fabricated by means of focused ion beam (FIB) from a $519 \mathrm{~nm}$, low pressure chemical vapor deposition (LPCVD) silicon nitride membrane. A detailed discussion of the reference cantilever measurement principle applied to the torsional system is also provided. Furthermore, the measurement of the micropaddle torsional spring constant is presented, where the torsional spring constant is determined by pushing a reference cantilever with known spring constant against the micropaddle, using AFM.

\section{Fabrication}

The micropaddle is fabricated from a $519 \mathrm{~nm}$ thick LPCVD silicon nitride membrane window (Silson Ltd., England), with a membrane size of $0.5 \mathrm{~mm} \times 0.5 \mathrm{~mm}$, which is supported by a $450 \mu \mathrm{m}$ thick silicon frame of size $7.5 \mathrm{~mm} \times 7.5 \mathrm{~mm}$. The Young's modulus of silicon nitride is determined to be $E=197 \pm 5 \mathrm{GPa}$ using nanoindentation technique [22] with assumption of the Poisson's ratio of $v=0.27$ [23]. The micropaddle pattern is fabricated by means of $\mathrm{Ga}^{+}$focused ion beam (FIB) milling, with an acceleration voltage of $30 \mathrm{keV}$ and beam current of $100 \mathrm{pA}$, using a

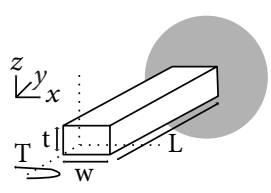

(a)

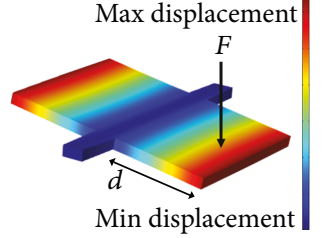

(b)
Figure 2: (a) A torsional beam and (b) schematic static response of the micropaddle when force $F$ is applied at a distance $d$ from the anchors.

FEI Strata DB-235 FIB-SEM dual beam tool. The dimensions of the micropaddle are $8 \mu \mathrm{m} \times 10 \mu \mathrm{m}$, and it is supported via two anchored beams along its length. The beam dimensions are $1 \mu \mathrm{m} \times 2.5 \mu \mathrm{m}$, and the anchors are placed either at the centre (represented by MP-1) or offset from the centre by $2.5 \mu \mathrm{m}$ (represented by MP-2) to give two different torsional system configurations (Figure 1).

\section{Theory and Measurement Principle}

In this study, an AFM cantilever (Nanosensors PPP-NCL), which was made of $\mathrm{Si}$ and presented a $10 \mathrm{~nm}$ radius of a curvature hemispherical tip, is used to measure the torsional spring constant of the micropaddle. This work was carried out using a NanoWizard II AFM (JPK Instruments, UK). Firstly, the spring constant of the AFM cantilever is calibrated using its resonance frequency, length, and width [21]. Finally, the paddle area of the micropaddle is divided into an array of $64 \times 64$ pixels, and the AFM cantilever is used to push the paddle at each pixel with $500 \mathrm{nN}$. The force spectroscopy data for all the points are saved and analysed to calculate the torsional spring constant using the following theory.

The micropaddle's beam can be considered as a cantilever with rectangular cross section, as shown in Figure 2(a). Applying the torsional moment $T$ on the beam's free end would force it to twist by an angle $\theta$, which is proportional to $T$ by means of the torsional spring constant $k_{p}$ $\left(k_{p}=T / \theta=G J / L\right)$, where $G=E /(2(1+v)), J$, and $L$ are the shear modulus, torsional constant, and the beam's length, respectively. The torsional constant $J$ for a rectangular cross section where the width, $w$, is greater than the thickness, $t$, can be expressed as follows [24]:

$$
J=\frac{w t^{3}}{3}\left[1-0.616\left(\frac{t}{w}\right)-0.087\left(\frac{t}{w}\right)^{2}+0.125\left(\frac{t}{w}\right)^{3}\right]
$$

Applying a force $F$ at a distance $d$ from the micropaddle's anchor along its length (Figure 2(b)) produces a torsional moment, and as a result, the micropaddle rotates by an angle $\theta_{p}$.

The two supporting beams on either side of the micropaddle act as two parallel springs; so, the effective torsional spring constant can be expressed by $K_{p}=2 k_{p}=$ 


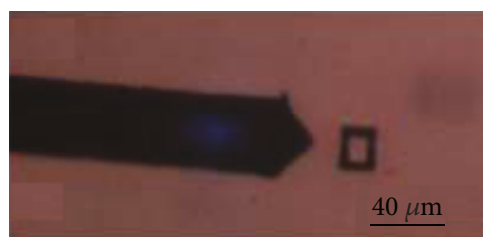

(a)

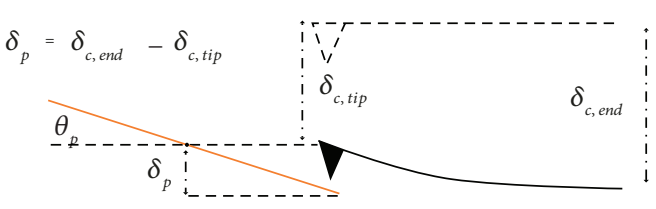

(b)

Figure 3: (a) AFM camera image of the micropaddle and the AFM probe. (b) Schematic of applying a torsional moment to the micropaddle using the AFM cantilever.

$1.95 \mathrm{nN} \cdot \mathrm{m}$ and is related to the torsional moment and angle of rotation as

$$
T=F d=K_{p} \theta_{p}
$$

Based on (1), the maximum angle of rotation and consequently displacement for the micropaddle occur when the force is applied at the furthest distance from its anchor (Figure 2(b)).

The substrate, with the micropaddle manufactured in its centre, was adhered to a glass microscope slide using a double-sided carbon sticky tape (Fisher Scientific Ltd., UK). The glass slide was immobilised onto a sample holder using spring clips. The sample holder is adhered to the AFM stage using a magnetically actuated three-point alignment system; the AFM camera image is shown in Figure 3(a). The AFM cantilever probe is mounted on a piezo stage and approaches the micropaddle from above. Consequently, the free end of the cantilever and the micropaddle will come into contact resulting in the application of a force to the micropaddle. As a result of the applied force, the AFM cantilever is deflected and the micropaddle undergoes an angular displacement due to the applied torsional moment around its beams. For very small angular displacement, it can be approximated as $\theta_{p} \cong \delta_{p} / d$ where $\delta_{p}$ is the micropaddle's free end displacement. The deflection of the AFM cantilever $\left(\delta_{c \text {,tip }}\right)$ is determined by the motion of the reflected laser beam onto the photodetector. Since the cantilever probe is fixed to the stage, the displacement of the fixed end is equal to the stage displacement $\left(\delta_{c, \text { end }}\right)$. The micropaddle's displacement $\left(\delta_{p}\right)$ at the point which is in contact with the AFM probe is deflected by an amount $\delta_{c \text {, end }}-\delta_{c \text {,tip }}$, as shown in Figure 3(b). The indentation depth on total displacement is negligible. Based on the following assumptions, Hertzian contact mechanics [25], considering $E_{\mathrm{Si}}=165 \mathrm{GPa}$ and $v_{\mathrm{Si}}=0.22$ [26] for the AFM cantilever, $E_{\mathrm{SiN}_{\mathrm{x}}}=197 \mathrm{GPa}$ and $v_{\mathrm{SiN}_{\mathrm{x}}}=0.27$ for the micropaddle and having the rigid substrate (micropaddle), the indentation depth would be $1.15 \mathrm{~nm}$ due to $500 \mathrm{nN}$ compressive load. However, the micropaddle is not fixed; hence, the indentation depth will be much less than $1.15 \mathrm{~nm}$, and its contribution to total displacement is negligible.

The force applied by the AFM cantilever is evaluated using its spring constant $k_{c}$ and tip deflection $\delta_{c}$, such that $F=k_{c} \delta_{c}$. Based on Newton's third law, the applied force value

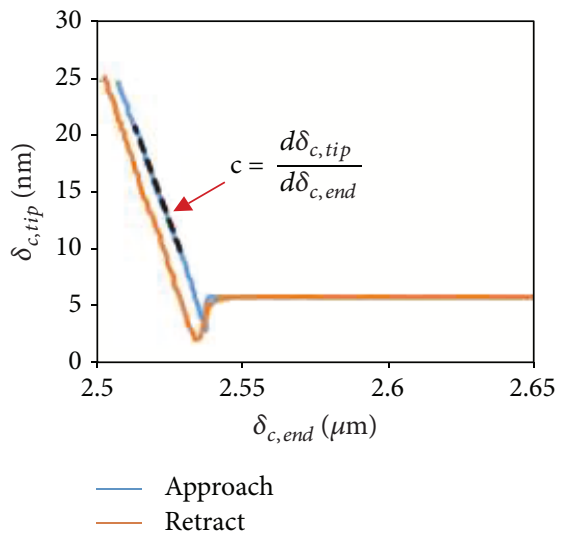

FIgURE 4: Example of AFM cantilever tip displacement, approach, and retract curve for a pixel on the micropaddle area.

is the same at the contact point for the micropaddle and cantilever.

$$
k_{c} \delta_{c, \text { tip }}=\frac{K_{p} \delta_{p}}{d^{2}}=\frac{K_{p}\left(\delta_{c, \text { end }}-\delta_{c, \text { tip }}\right)}{d^{2}} .
$$

Hence, the torsional spring constant $K_{p}$ can be calculated using the following:

$$
\begin{gathered}
K_{p}=k_{c} d^{2} \frac{c}{1-c}, \\
c=\frac{d \delta_{c, \text { tip }}}{d \delta_{c, \text { end }}},
\end{gathered}
$$

where $c$ is the slope of the cantilever tip deflection $\left(\delta_{c \text {,tip }}\right)$ versus measured height $\left(\delta_{c, \text { end }}\right)$ during approach/retract and is unitless (Figure 4). In this work, the value of $c$, used for calculation of the torsional spring constant, is set as the average slope during approach and retract. This average value is taken so as to reduce the influence of friction between the cantilever tip and the micropaddle [27]. The misalignment of the approach and retract curves is an artefact of the measurement, due to hysteresis in the piezoelectric scanner when the direction of travel is reversed.

\section{Results and Discussion}

In the cantilever reference method, the AFM cantilever is tilted by an angle $\phi$, which is the angle between the cantilever and the horizontal axis. As the optical lever is measuring the 


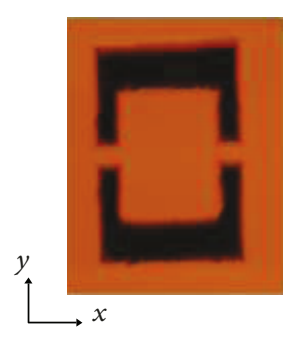

(a)

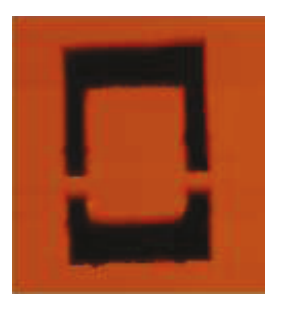

(b)
Figure 5: AFM images $(\sim 64 \times 64$ pixels $)$ of the micropaddle ( $x$-width $=8 \mu \mathrm{m}, y$-length $=10 \mu \mathrm{m}$ ). (a) MP-1. (b) MP-2.

vertical deflection of the AFM cantilever, the effective spring constant $\left(k_{\text {effective }}=k_{c} / \cos ^{2}(\phi)\right)$ which is the perpendicular stiffness to the surface of the micropaddle needs to be used instead of its intrinsic spring constant [13]. In this work, $\phi$ and $k_{\text {effective }}$ are 10 degree and $27.65 \mathrm{~N} \mathrm{~m}^{-1}$.

It is worth noting that the effect of sample tilt on the measurements is negligible. The NanoWizard II AFM is a stateof-the-art measurement system, with repeatable tip/sample alignment due to the magnetically actuated three-point alignment system and the greatest source of tilt error will be due to the double-sided carbon sticky tape. Given the tape thickness $(90 \mu \mathrm{m})$ and the sample dimensions $(7.5 \mathrm{~mm} \times 7.5 \mathrm{~mm})$, the highest possible tilt from the horizontal is expected to be less than 1 degree, which would have a negligible $(<1 \%)$ impact on the data.

AFM images of the micropaddles are shown in Figure 5. JPK Data Processing software (JPK Instruments, UK) is used to measure and extract the force spectroscopy data for all scanned points on the micropaddle surface. A bespoke MATLAB code is developed to analyse the force spectroscopy data for each individual pixel. The slope $c$ for all the pixels is calculated and presented in Figure 6.

The slope along the width almost remains constant which suggests that the micropaddle's displacement is the same and almost independent of the position of the applied force along its width (Figure 6(c)). Figure 6(d) presents the slope for MP1 along its length which is symmetrical around the centre. As the AFM cantilever scans the micropaddle along its length, the slope increases from one free end to the centre of rotation and decreases again when it moves away from the centre and the minimum and maximum slopes occur at its free ends and centre of rotation.

In the theoretical calculation, the beams' anchors are fixed and when the AFM cantilever is pushed against the beam (i.e., on the torsional axis), almost no deflection is expected in the $z$-direction. This is due to the beams' dimensions and the resultant spring constant in $z$-direction $K_{z z, p}$, as $K_{z z, p}=2 \times\left(12 E I_{x} / L^{3}\right) \approx 3500 \mathrm{~N} \mathrm{~m}^{-1}$, which is two orders of magnitude greater than the spring constant of the AFM cantilever $\left(27.65 \mathrm{~N} \mathrm{~m}^{-1}\right)$.

If the anchors were fixed, the theoretical slope should be equal to 1 through the centreline passing through the two anchors, which means that the displacement of the cantilever tip would be equal to its end displacement. However, the anchors are found not to be completely fixed as the empirically achieved slope is $\sim 0.7$ which indicates that the micropaddle undergoes some vertical displacement in this region.

The torsional spring constant should remain the same through the length of the micropaddle centreline and is independent from the position where the force is applied. The theoretical slope is calculated by substituting the theoretical torsional spring constant $\left(K_{p}=2 G J / L=1.95 \mathrm{nN} \cdot \mathrm{m}\right)$ of the micropaddle into (4).

The theoretical slope for MP-1 is shown in Figure 7(a). As it is presented, the slope from experiment follows the same trend, but it is offset by the difference between the maximum theoretical slope through calculation and experimental slope. The experimental values are transformed by adding the offset value to the measured experimental slope. As it is illustrated in Figure 7(a), the transformed values are in good agreement with theoretical values. The micropaddle is fabricated at the centre of a $519 \mathrm{~nm}$ thick, $500 \mu \mathrm{m} \times 500 \mu \mathrm{m}$ silicon nitride membrane, which is supported by a silicon frame and so is not a rigid area. The extra displacement for the micropaddle can be explained by the deflection of this membrane window.

However, for the micropaddle where the anchors are offset by $2.5 \mu \mathrm{m}$ (MP-2), the transformed slope does not completely fit the theoretical slope even after applying the offset, as shown in Figure 7(b). The effect starts to be greater for distances larger than $5 \mu \mathrm{m}$ from the anchor. In fact, as the distance from the anchor increases, the effect of membrane deflection reduces, since torsion becomes the dominant effect on the anchors, and so the offset does not need to be applied uniformly to all of the measured data. The effect is governed by the micropaddle torsional spring rigidity and the silicon nitride membrane compliance. It is worth noting that the effect of unbalanced weight for MP-2 on the measurements can be neglected. The mass and centre of mass for the unbalanced part are $62.28 \mathrm{fg}$ (assuming silicon nitride density $=3000 \mathrm{~kg} / \mathrm{m}^{3}$ [23]) and at $5 \mu \mathrm{m}$ offset from the anchors. This would consequently lead to $3.05 \mathrm{aN} \cdot \mathrm{m}$ torque on the torsional springs. The minimum and maximum torques due to the force from the AFM probe $(500 \mathrm{nN})$ on the unbalanced part at corresponding offset distance of $2.5 \mu \mathrm{m}$ and $7.5 \mu \mathrm{m}$ from the centre of rotation are $1.25 \mathrm{pN} \cdot \mathrm{m}$ and $3.75 \mathrm{pN} \cdot \mathrm{m}$ which are almost six orders of magnitude greater compared to the added torque due to the unbalanced weight on the torsional springs. Therefore, the effect of unbalanced weight compared to the AFM probe force on the measurements is negligible.

If the nontorsional spring constant $K_{z z, p}$ of the anchors of the micropaddle was in a comparable range as the AFM cantilever spring constant, then the slope would also be a function of distance based on the relative values of $K_{p}, K_{z z, p}$ and the membrane compliance.

For MP-1 presented here, the mean torsional spring constant along the length for $d>1.5 \mu \mathrm{m}$ is evaluated and is shown to be equal to $1.84 \pm 0.1 \mathrm{nN} \cdot \mathrm{m}$ (Figure $8(\mathrm{a})$ ). The experimental (after offsetting data) and theoretical torsional spring constants of the micropaddle are in good agreement, and the error is $\sim 6 \%$. The torsional spring constants for 
Average slope for approach and retract (MP-1)

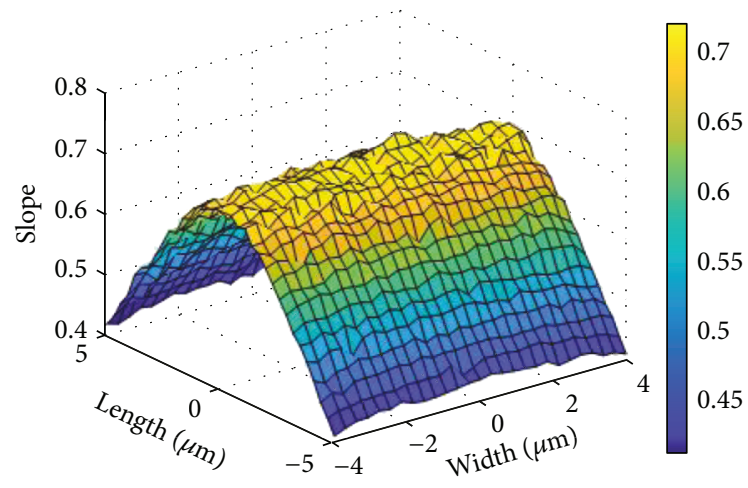

(a)

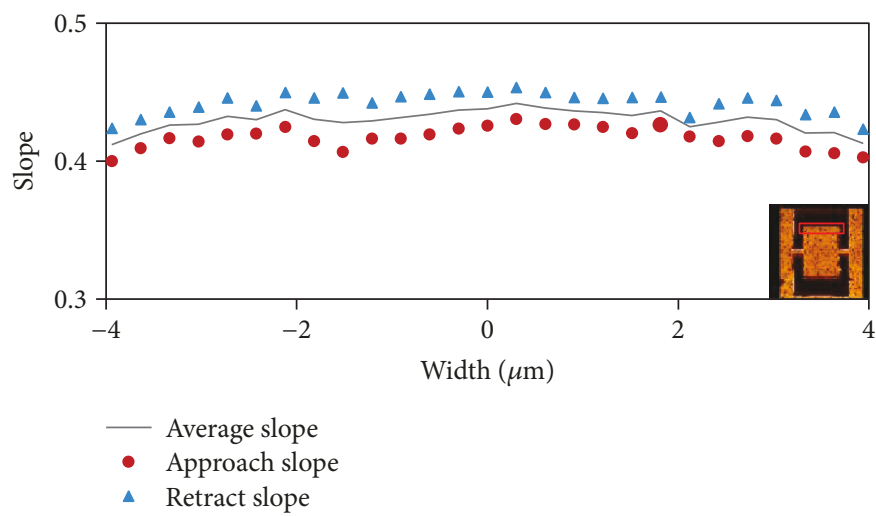

(c)
Average slope for approach and retract (MP-2)

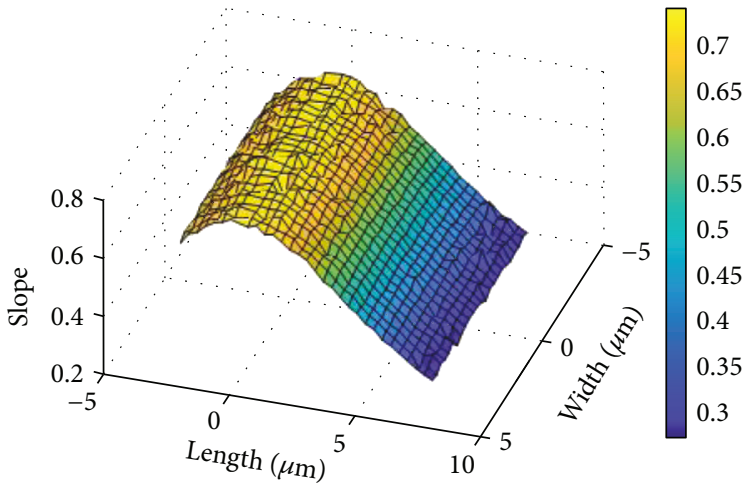

(b)

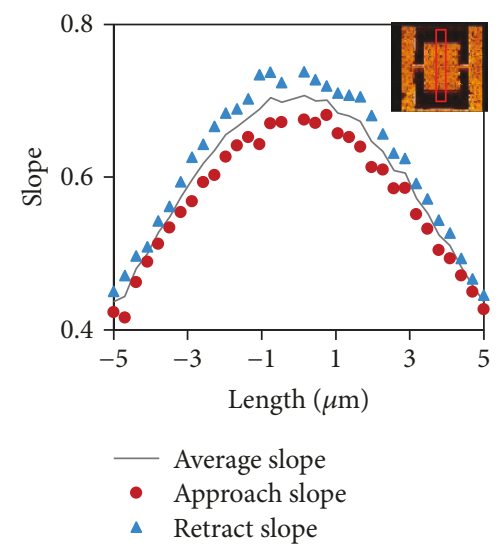

(d)

Figure 6: $8 \mu \mathrm{m} \times 10 \mu \mathrm{m}$ micropaddle's map of the slope over the surface for (a) MP-1 and (b) MP-2. (c) Slope $c$ along the width at the furthest edge from the anchor for MP-1. (d) Slope $c$ along the length at centre for MP-1.

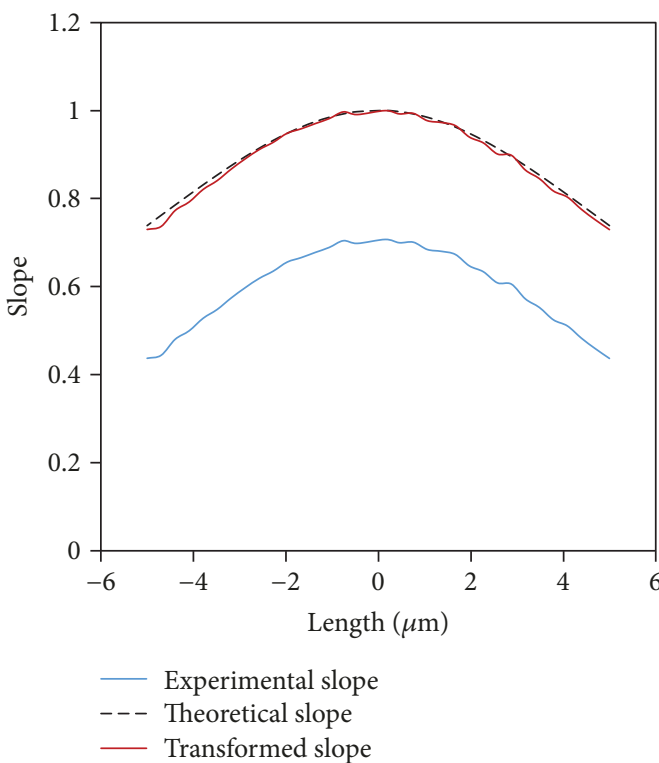

(a)

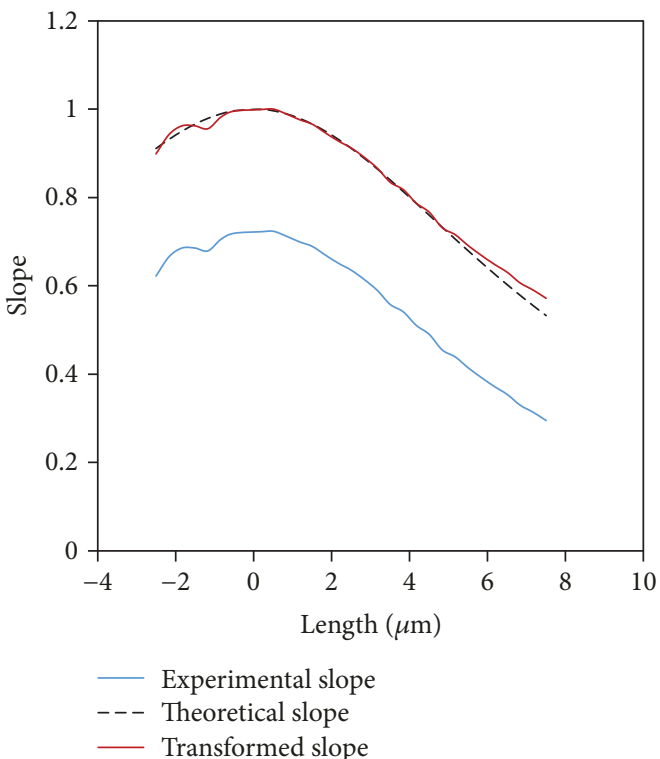

(b)

FIgURE 7: Slope $c$ along the centreline of the micropaddle (a) for MP-1 and (b) for MP-2. 


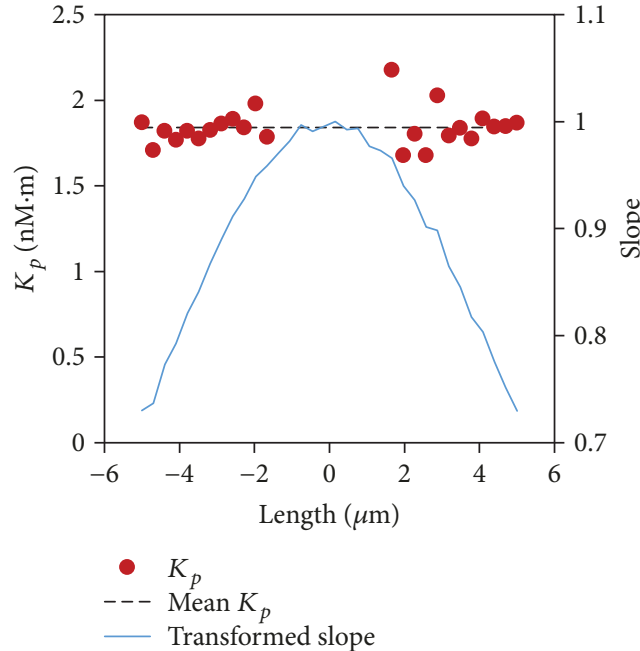

(a)

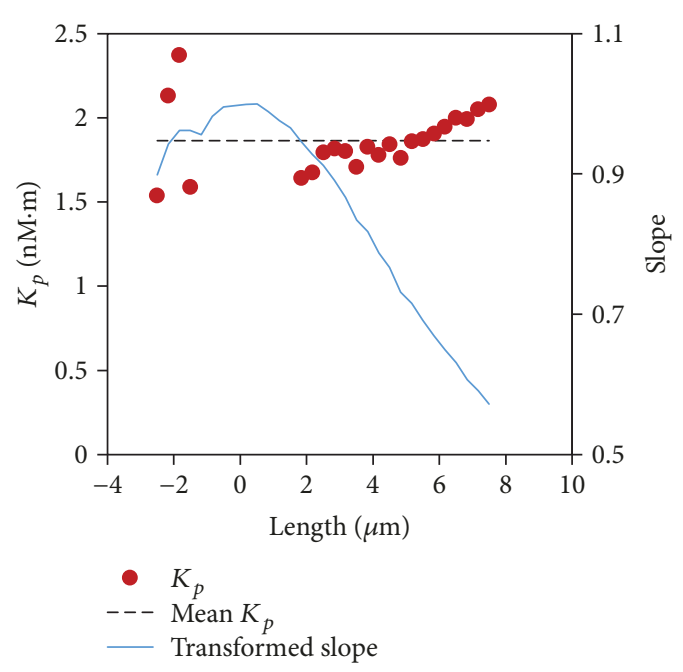

(b)

FIGURE 8: Transformed slope $c$ and corresponding torsional spring, $K_{p}(\mathrm{nN} \cdot \mathrm{m})$, along the centreline of the micropaddle (a) for MP-1 and (b) for MP-2.

MP-2 should be the same as MP-1 since they have the same beam dimensions, are made of same material, and are fabricated by the identical technique. Transferring the data by the offset value for MP-2 and calculating the mean torsional spring, $1.86 \pm 0.2 \mathrm{nN} \cdot \mathrm{m}$, along the length for $d>1.5 \mu \mathrm{m}$ result in $\sim 5 \%$ error (Figure $8(\mathrm{~b})$ ). At $d>5 \mu \mathrm{m}$, where the transformed experimental slope and theoretical slope are not identical, as shown in Figure $7(\mathrm{~b})$, the experimental torsional spring constant is seen to deviate in a linear manner from the values below $d=5 \mu \mathrm{m}$ (Figure $8(\mathrm{~b})$ ). The difference between the experimental slope and theoretical slope can be fitted linearly; therefore, the effect of membrane compliance on the experimentally observed total displacement of the micropaddle is reduced linearly at $d>5 \mu \mathrm{m}$ for this particular micropaddle.

\section{Conclusions}

In this study, the torsional spring constant of the micropaddles is shown to be able to be determined by the reference cantilever method using AFM force spectroscopy. The micropaddles are fabricated out of $519 \mathrm{~nm}$ thick silicon nitride membrane using FIB. The paddle area imaged by the AFM is divided into a grid of $64 \times 64$ pixels, with forcedisplacement data acquired at all points. The gradient of the force curve is used to find the mean torsional spring constant at distances greater than $1.5 \mu \mathrm{m}$ from the anchors. The differences from calculated values are found to be less than $6 \%$ for both micropaddles. As the anchors are not completely fixed, larger displacement compared to theory for the paddle rotation is observed which is due to the silicon nitride membrane deflection; however, the same theoretical gradient trend is observed. This indicates that the membrane deflects similarly through the micropaddle area for micropaddle with anchors at the centre. For the case when the micropaddle is part of another suspended membrane, the displacement can be considered as a function of distance from the centre and is dependent on membrane compliance and the anchors' normal and torsional spring constant. The suitability of this method to characterize torsional microsystems has been shown, and it is clear that it can be applied to the characterization and understanding of the mechanical behaviour of micromirrors or similar structures.

\section{Data Availability}

The data used to support the findings of this study are available from the corresponding author upon request.

\section{Conflicts of Interest}

The authors declare that there is no conflict of interest regarding the publication of this paper.

\section{Acknowledgments}

The Micro Materials NanoTest and JPK Instruments NanoWizard II AFM used in this research were obtained through Birmingham Science City: Innovative Uses for Advanced Materials in the Modern World (West Midlands Centre for Advanced Materials Project 2), with support from Advantage West Midlands (AWM) and part funded by the European Regional Development Fund (ERDF).

\section{References}

[1] G. Silva, F. Carpignano, F. Guerinoni, S. Costantini, M. de Fazio, and S. Merlo, "Optical detection of the electromechanical response of MEMS micromirrors designed for scanning picoprojectors," IEEE Journal of Selected Topics in Quantum Electronics, vol. 21, no. 4, pp. 147-156, 2015.

[2] X. C. Zhang, E. B. Myers, J. E. Sader, and M. L. Roukes, "Nanomechanical torsional resonators for frequency-shift infrared thermal sensing," Nano Letters, vol. 13, no. 4, pp. 1528-1534, 2013. 
[3] J. Jeong, S. Kumagai, I. Yamashita, Y. Uraoka, and M. Sasaki, "Micromechanical IR thermal detector using torsional oscillation: improvement of resonator profile for high sensitivity," Japanese Journal of Applied Physics, vol. 54, no. 4S, article 04DE07, 2015.

[4] R. P. Manginell, D. R. Adkins, M. W. Moorman et al., "Masssensitive microfabricated chemical preconcentrator," Journal of Microelectromechanical Systems, vol. 17, no. 6, pp. 13961407, 2008.

[5] J. Haneveld, D. M. Brouwer, A. Mehendale et al., "MEMSbased micro-coriolis mass flow sensor," in Proceedings of the 10th Anniversary International Conference of the European Society for Precision Engineering and Nanotechnology, vol. 2, pp. 560-564, Zürich, May 2008.

[6] N. Mahmoodi, Design and Development of MEMS Biosensors, University of Birmingham, 2017.

[7] B. Boonliang, P. D. Prewett, J. Hedley, J. Preece, and C. A. Hamlett, "A focused-ion-beam-fabricated micro-paddle resonator for mass detection," Journal of Micromechanics and Microengineering, vol. 18, no. 1, article 015021, 2007.

[8] G. Binnig, C. F. Quate, and C. Gerber, "Atomic force microscope,” Physical Review Letters, vol. 56, no. 9, pp. 930-933, 1986.

[9] J. P. Cleveland, S. Manne, D. Bocek, and P. K. Hansma, "A nondestructive method for determining the spring constant of cantilevers for scanning force microscopy," Review of Scientific Instruments, vol. 64, no. 2, pp. 403-405, 1993.

[10] J. M. Neumeister and W. A. Ducker, "Lateral, normal, and longitudinal spring constants of atomic force microscopy cantilevers," Review of Scientific Instruments, vol. 65, no. 8, pp. 2527-2531, 1994.

[11] T. Senden and W. Ducker, "Experimental determination of spring constants in atomic force microscopy," Langmuir, vol. 10, no. 4, pp. 1003-1004, 1994.

[12] A. Torii, M. Sasaki, K. Hane, and S. Okuma, "A method for determining the spring constant of cantilevers for atomic force microscopy," Measurement Science and Technology, vol. 7, no. 2, pp. 179-184, 1996.

[13] M. Tortonese and M. Kirk, "Characterization of applicationspecific probes for SPMs," in Photonics West'97, International Society for Optics and Photonics, 1997.

[14] C. A. Clifford and M. P. Seah, "The determination of atomic force microscope cantilever spring constants via dimensional methods for nanomechanical analysis," Nanotechnology, vol. 16, no. 9, pp. 1666-1680, 2005.

[15] C. A. Clifford and M. P. Seah, "Improved methods and uncertainty analysis in the calibration of the spring constant of an atomic force microscope cantilever using static experimental methods," Measurement Science and Technology, vol. 20, no. 12, article 125501, 2009.

[16] R. S. Gates and M. G. Reitsma, "Precise atomic force microscope cantilever spring constant calibration using a reference cantilever array," Review of Scientific Instruments, vol. 78, no. 8, article 086101, 2007.

[17] D. S. Golovko, T. Haschke, W. Wiechert, and E. Bonaccurso, "Nondestructive and noncontact method for determining the spring constant of rectangular cantilevers," Review of Scientific Instruments, vol. 78, no. 4, article 043705, 2007.

[18] H. -J. Butt and M. Jaschke, "Calculation of thermal noise in atomic force microscopy," Nanotechnology, vol. 6, no. 1, pp. 1-7, 1995.
[19] I. M. Malovichko, "Measuring AFM cantilever stiffness from a thermal noise spectrum," Bulletin of the Russian Academy of Sciences: Physics, vol. 77, no. 8, pp. 972-974, 2013.

[20] R. T. Howe, "Polycrystalline silicon micromachining: a new technology for integrated sensors," Annals of Biomedical Engineering, vol. 14, no. 2, pp. 187-197, 1986.

[21] J. Bowen, D. Cheneler, D. Walliman et al., "On the calibration of rectangular atomic force microscope cantilevers modified by particle attachment and lamination," Measurement Science and Technology, vol. 21, no. 11, article 115106, 2010.

[22] A. C. Fischer-Cripps, Nanoindentation of Thin Films and Small Volumes of Materials, in Nanoindentation, Springer, 2011.

[23] H. O. Pierson, Handbook of Chemical Vapor Deposition: Principles, Technology and applications, William Andrew, 1999.

[24] K. B. Lee, Principles of Microelectromechanical Systems, John Wiley \& Sons, 2011.

[25] K. Johnson, Contact Mechanics, Cambridge University Press, Cambridge, UK, 1985.

[26] J. Dolbow and M. Gosz, "Effect of out-of-plane properties of a polyimide film on the stress fields in microelectronic structures," Mechanics of Materials, vol. 23, no. 4, pp. 311321, 1996.

[27] J. R. Pratt, G. A. Shaw, L. Kumanchik, and N. A. Burnham, "Quantitative assessment of sample stiffness and sliding friction from force curves in atomic force microscopy," Journal of Applied Physics, vol. 107, no. 4, article 044305, 2010. 


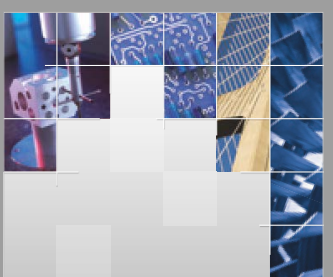

\section{Enfincering}
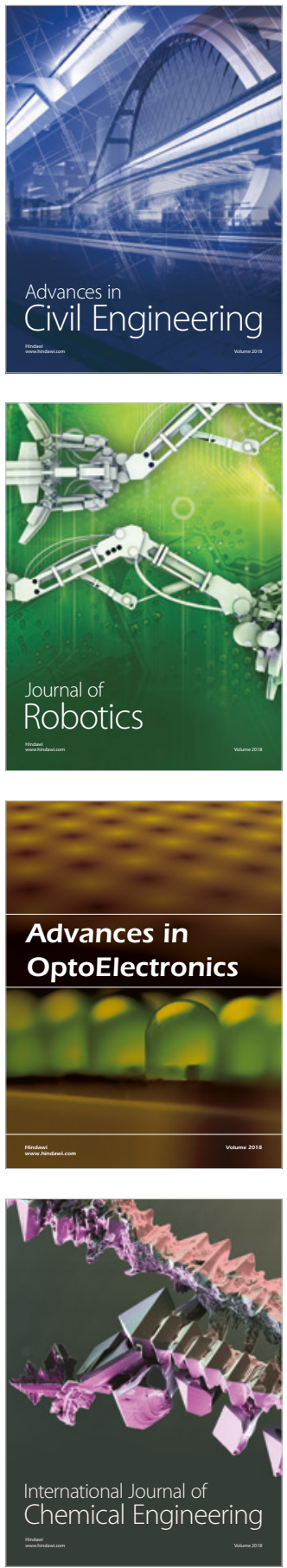

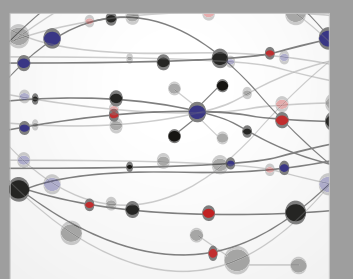

\section{Rotating \\ Machinery}

The Scientific World Journal

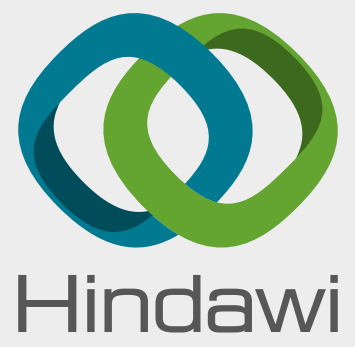

Submit your manuscripts at

www.hindawi.com
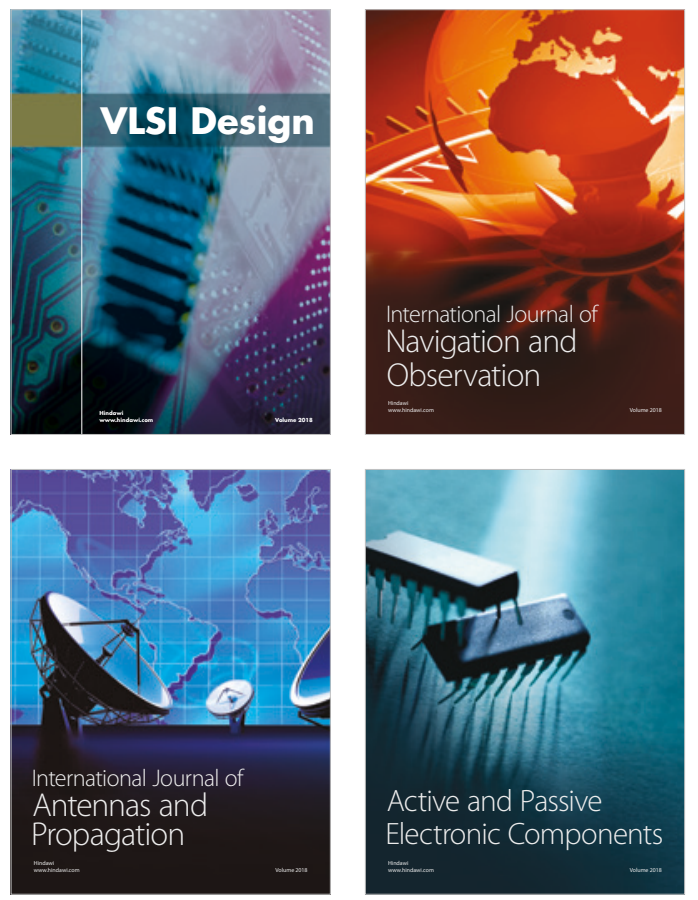
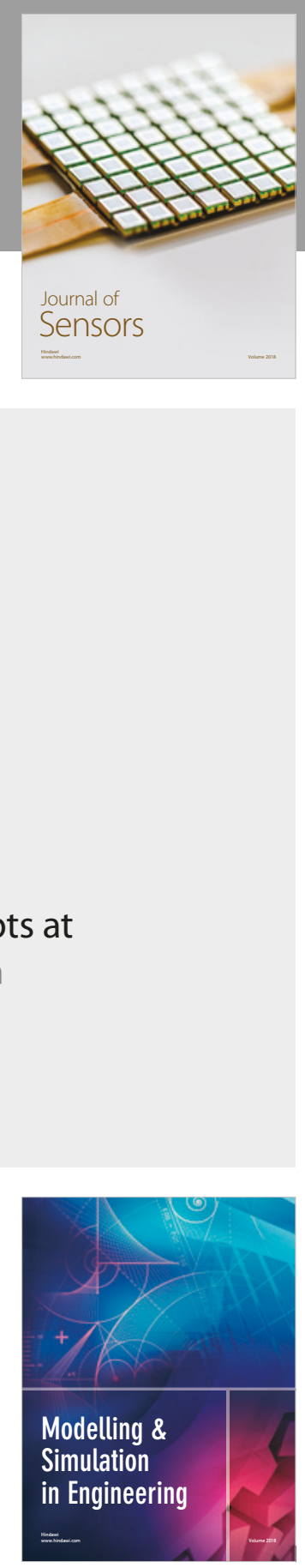

\section{Advances \\ Multimedia}
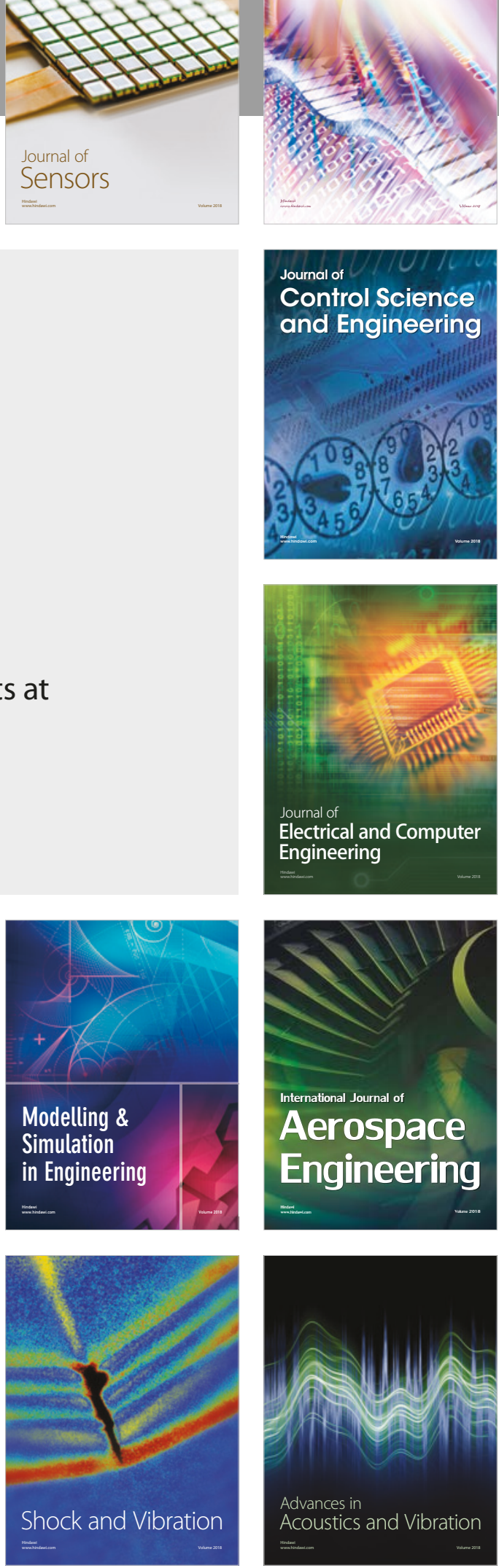